\title{
Comparaison de trois méthodologies ESG : les bases de données extra-financières fournissent-elles la même information?
}

\author{
Frédérique Bardinet-Evraert Maître de Conférences \\ IAE Bordeaux Université de Bordeaux (France)
}

L'objet de la recherche est d'étudier la convergence des notes ESG de trois organismes de notation extra-financière. Dans un contexte de tétranormalisation (Savall et Zardet, 2005), la validité et la conformité des ratings ESG, compte tenu des missions confiées aux agences, sont peu étudiées (Chatterji et al., 2016). L'étude porte sur un échantillon de 247 sociétés cotées européennes et 65 anglo- saxonnes lors des années 2009-2012. Elle évalue les différences d'approche des trois organismes. La méthodologie utilisée est l'analyse en composantes principales qui établit les corrélations entre les scores puis la régression longitudinale qui identifie les facteurs de différenciation observés.

Mots-clés : Agences de notation - Scores environnemental - Social et gouvernance - Tétranormalisation.

The aim of the research is to study the convergence of the ESG ratings of three non-financial rating organizations. In the context of tetrastandardization (Savall and Zardet, 2005), the validity and conformity of ESG ratings, given the missions entrusted to the agencies, are poorly studied (Chatterji et al., 2016). The study is based on a sample of 247 listed European companies and 65 Anglo-Saxons in the years 2009-2012. It assesses the differences in approach of the three organizations. The methodology used is the principal component analysis which establishes the correlations between the scores and then the longitudinal regression which identifies the differentiation factors observed.

Key-Words: Rating agencies - Environmental - Social and governance scores - Tetra-standardization. 
El objetivo de esta investigación es estudiar la convergencia de las calificación de ESG (medioambiente; lo social, y el gobierno) de tres organismos de notación extra financieros. En un contexto de tetra normalización (Savall y Zardet, 2005), la validez y la conformidad del rating ESG, teniendo en cuenta la misión confiada a los agentes, son poco estudiadas (Chatterji, 2016). El estudio está basado en una muestra de 247 empresas cotizadas europeas y 65 anglosajonas durante los años 2009 y 2012. En él se evalúa los diferentes enfoques de tres organismos. La metodología utilizada es el análisis en componentes principales que establecen las correlaciones entre la puntuación, y además, la regresión longitudinal que identifica los factores de la diferenciación observada.

Palabras-clave: Agencia de calificación - Puntuación medioambiental - Social y gobierno - Tetra normalización.

\section{Introduction}

La mesure par ratings de la performance environnementale, sociale et de gouvernance est un enjeu majeur avec le développement de l'utilisation de ces informations par les acteurs économiques comme pour la performance économique des entreprises. Une des voies pour percevoir le niveau de responsabilité des entreprises est de se référer aux notes ESG publiées par des organismes indépendants. Leur mission est de réduire l'asymétrie d'information entre les investisseurs, les entreprises et les parties prenantes.

Les managers, les dirigeants et les investisseurs utilisent désormais les ratings non financiers pour leurs décisions stratégiques (Chatterji et al., 2009). Presque tous supposent l'homogénéité et la validité des notes publiées par les agences. Or, les ratings utilisés pour estimer la performance ESG font rarement l'objet d'une évaluation ou d'un audit. Les méthodologies et les processus de notation manquent de transparence. Les managers et les dirigeants consacrent de plus en plus de ressources et de temps pour les activités RSE: plus de 8000 entreprises ont signé l'UN Global Compact comme signe d'engagement dans la RSE. Les investisseurs utilisent les ratings pour leurs arbitrages sur les marchés financiers. 
Ils doivent être capables de distinguer les entreprises socialement responsables des autres. Des notes erronées peuvent les abuser et conduire à des allocations de fonds sous optimales. Les enjeux financiers sont importants avec plus de $15 \%$ des investissements européens placés dans des fonds ISR (Eurosif, 2014). Pour les académiques, les recherches en la matière ont été très riches ces 20 dernières années (Orlitzky, Schmidt, \& Rynes, 2003) et s'appuient dans leur grande majorité sur les notes ESG fournies par des agences, comme KLD, MSCI, EIRIS, ETIX, Sustainanalytics, VIGEO ou ASSET 4. Les notes de ces organismes sont utilisées dans les recherches, mais peu d'entre-elles s'intéressent directement à la transparence, à la précision des ratings et à la convergence de leurs résultats (Sharfman, 1996, Igalens, 2005, Delmas, 2013, Chatterji et al., 2016). La part variable des rémunérations des dirigeants et des administrateurs est indexée sur des critères ESG. Il apparait donc utile de comprendre la variété et les contextes qui favorisent la convergence des évaluations.

La mesure de la performance ESG à travers un score est ambigüe en raison du nombre de définitions et de conceptions différentes. S'il existe de nombreuses définitions de la performance RSE (Caroll, 1979, Wartick et al.,1985, Wood , 1991, Clarkson, 1995), on dénombre presque autant d'approches pour la mesure : évaluation par des experts du secteur, utilisation d'indicateurs multiples, enquêtes auprès des managers et des dirigeants (Graafland et al., 2008 ; Maignan and Ferrell, 2003), mesures spécifiques des ONG et des groupes de lobbying, investisseurs pour la prévision financière (Marquez et Fombrun, 2005). Pour les organismes de notation, c'est un facteur de différenciation, chacun ayant sa propre définition, son propre modèle, ses outils et méthodes de mesure. La grande latitude laissée aux organismes de notation extra-financière pose la problématique de la comparabilité des scores obtenus.

La recherche se propose d'apprécier si les ratings environnementaux, sociaux et de gouvernance de trois organismes spécialisés dans la notation, pour le même échantillon d'entreprises, convergent. L'étude est complétée par l'examen des facteurs économiques et institutionnels capables d'influencer les scores obtenus.

La première partie présente le cadre théorique de référence et les hypothèses. La seconde partie décrit la méthodologie, l'échantillon, les variables, la méthode et les résultats obtenus. La troisième partie 
est consacrée à la discussion des résultats.

\section{1.- Le cadre théorique et les hypothèses de recherche}

\subsection{Multiplication des normes, contraintes et tétranormalisation}

L'étude se situe principalement dans le contexte de l'Union Européenne. Au sein de celle-ci, les mesures produites par les agences de notation ESG se sont développées à partir des années 2000 sous l'effet d'incitations gouvernementales, de codes et de principes. En fait, l'Europe a imposé de nombreux référentiels à travers le monde et est souvent considérée comme pionnière en matière de RSE. Selon l'index de performance environnementale calculé par des chercheurs de l'Université de Yale et de Colombia, 18 des 20 pays du classement 2012 sont européens. Depuis la loi NRE de 2001, des principes, règles et normes sur la RSE n'ont cessé d'être proposés, comme par exemple les principes pour l'investissement responsable (2006), le code de transparence d' Eurosif (2008, 2013), la loi Grenelle II § 224 qui exige des entreprises d'investissement l'utilisation d'une déclaration obligatoire sur l'utilisation des critères ESG (France, 2011), la directive européenne sur la divulgation d'informations non financières (2014), la loi française de transition énergétique (2015§173) qui étend l'obligation de reporting des entreprises cotées aux entreprises de plus de 500 salariés et $200 \mathrm{M}$ d'euros de chiffre d'affaires. A côté des obligations légales, des organisations non gouvernementales, comme le Global Reporting Initiative, visent à promouvoir le développement des rapports RSE, ainsi que l'IIRC (2012), le comité international de l'information intégrée qui offre un référentiel permettant aux entreprises de gérer les risques ESG et d'établir des stratégies de long terme. Enfin, la norme IS0 26000 est un guide d'objectifs et de bonnes pratiques qui vise à clarifier la terminologie de la RSE, et permet de traduire ses principes en actes. En définitive, ces 20 dernières années ont été marquées par une prolifération de normes qui s'inscrivent dans un contexte plus large de tétranormalisation. Ce concept proposé par Savall et Zardet (2005), considère quatre domaines comme très normés dans l'environnement des entreprises : les normes relatives à la qualité, à la sécurité et l'environnement (ISO), celles relatives à la 
comptabilité financière et la gouvernance (IFRS), celles relatives au commerce (OMC) et enfin la norme relative au domaine social et administratif (Pacte mondial, OTT). La multiplication des normes conduit à des surenchères et à l'incohérence ce que précisément tout processus de normalisation devrait éviter, à leur application partielle ou encore à leur détournement et ne peut qu'aboutir à terme à leur perte de crédibilité d'où le désengagement des Etats par la délégation au marché, à des institutions de proximité et aux partenariats public-privé (Savall et Zardet, 2005).

En favorisant le développement des normes, les régulateurs ont de fait confié la notation ESG à des organismes indépendants. Cette logique de délégation du contrôle a eu tendance à déresponsabiliser les investisseurs comme les régulateurs et installé les agences dans une situation de rente. Le secteur étant relativement concentré et oligopolistique, cette situation peut apparaitre comme dangereuse. Pour la tétranormalisation, la transparence affichée d'une norme est jugée suspecte, les normes étant définies par les circonstances de leur mesure. Elles ne sont pas des objets abstraits indépendants séparés d'une manière ou d'une autre de leurs conditions matérielles (Cappelletti, 2008). Les agences se voient exercer une nouvelle mission de normalisation des fonds socialement responsables en offrant des références pour appréhender les caractéristiques ESG des entreprises. Comme le souligne Jacques Bichot (2015), la normalisation galopante impose aux entreprises d'innover dans la contrainte, d'anticiper les normes, de rester en conformité pour assurer leur survie. Si la tétranormalisation a permis l'installation d'un très grand nombre d'organismes indépendants en situation de quasi- régulateurs, elle oblige les dirigeants à respecter une multitude de normes, de règlements, de lois et de bonnes pratiques, parfois contradictoires, qui rendent de plus en plus complexe et coûteuse la gouvernance des entreprises (Cappelletti, 2008). L'inflation normative a conduit au développement d'organismes indépendants publiant de l'information et inventant leur propre cadre de mesure (Boje et Rosile, 2010). Selon Savall et Zardet (2005), les institutions publiques et privées augmentent de plus en plus leur offre concurrentielle de normes. Or, celles-ci ne sont pas seulement de saines règles du jeu, facteur d'équité, elles ont une face cachée : l'édification de barrières à l'entrée dans le jeu concurrentiel. Et comme les organismes, comme les individus, réagissent aux règles sur la base desquelles ils sont jugés, l'afflux de 
normes est aussi source d'uniformité et de risques. Le premier d'entre eux est celui de l'utilisation de la norme comme outil de marketing (Barth, 2009) ou de légitimation. La tétranormalisation soulève encore les questions de l'application effective des normes, de la réalité des sanctions et de la fraude (Zardet et Bonnet, 2010).

\subsection{Le marché des organismes de notation et les hypothèses de recherche}

Malgré l'existence de référentiels tels que la Global Reporting Initiative, les principes de l'OCDE (2006), la norme ISO 26000 , la notation des « critères RSE » intègre de nombreux enjeux dont le périmètre varie selon les organismes.

Le marché des agences de notation qui évaluent et notent les pratiques environnementales, sociales et de gouvernance s'est considérablement structuré depuis une quinzaine d'années à travers le monde. Ces agences se fondent sur des sources d'information multiples et utilisent chacune des critères d'évaluation spécifiques, en fonction de leur propre définition de la RSE, les informations étant parfois limitées ou influencées par le pays d'origine. Alors que les agences de notation exigent de la transparence de la part des entreprises, leurs méthodes d'évaluation restent opaques. C'est un secteur très concentré. Trois grandes familles d'acteurs se partagent le marché de la notation extra- financière (Novethic, 2014) : les fournisseurs de données financières et extra-financières comme ASSET 4 qui offrent des bases de données mondiales d'information ESG en plus des informations financières, les grandes agences de notations extra-financières à portée internationale comme VIGEO et des brokers spécialisés qui proposent des analyses extra-financières en créant de nouvelles équipes dédiées à la notation ESG.

Deux agences de notation souvent citées ont été sélectionnées : ASSET 4 et VIGEO qui occupent une place importante dans le secteur de la notation extra-financière et une société de gestion d'actifs spécialisée dans les fonds ISR qui a mis à notre disposition son fichier, mais qui ne souhaite pas être directement citée. Les trois organismes présentent des similitudes et des différences. Ils ont été créés au cours des 10-15 dernières années, procèdent à une collecte d'informations multi sources et notent des PME et des grandes entreprises des pays développés. Les méthodologies diffèrent par le 
champ couvert, plusieurs milliers d'entreprises mondiales pour les deux premières, ASSET 4 et VIGEO qui se focalisent sur l'ensemble des parties prenantes, facturent leurs services et utilisent entre 250 et 330 critères contre 80 pour la troisième laquelle réserve ses notations à ses seuls gérants de fonds et couvre près de mille entreprises principalement européennes. VIGEO a développé un référentiel et une méthodologie de notation originale, déclinés à travers une gamme de produits et services ESG destinés aux investisseurs et elle intervient aussi auprès des équipes et des managers pour intégrer les critères ESG dans les fonctions de l'entreprise. Elle note leur performance sur 38 enjeux ESG contextualisés et pondérés regroupés en 6 domaines et analyse les risques de réputation, humain, juridique et opérationnel associés. VIGEO respecte la norme Arista 3.0, norme qui favorise la qualité, l'intégrité et la transparence des agences de recherche extrafinancière. ASSET4 est un fournisseur de données brutes qui intègre de l'information financière et de l'information non financière (Thomson Reuters). C'est la première agence à se lancer avec un positionnement de fournisseur d'informations ESG brutes utilisables par les investisseurs. L'évaluation qui comprend 250 indicateurs clés et 750 points de données conduit à un score global, la note de chaque point de données pouvant être fournie aux clients. «ASSET 4 offre une information objective pertinente et systématique sur l'environnement, le social et la gouvernance, afin que les investisseurs aient un large choix de stratégies d'investissement ISR ». Pour la société de gestion d'actifs, l'équipe se compose de 20 gérants et de 4 analystes ESG chargés d'évaluer les entreprises et disposant de plusieurs antennes en Europe. Quatre-vingt critères font l'objet d'un scoring sur les trois axes : l'environnement, le social, et la gouvernance. La société de gestion d'actifs cherche à se différencier de ses concurrents directs par la qualité de ses notes ESG réalisées en interne.

Le scoring des agences a un caractère multidimensionnel. Les données sont produites par un organisme externe aux entreprises et elles utilisent des sources de données diversifiées. Cependant, l'analyse porte sur des données agrégées. Les référentiels ne reposent pas sur un modèle théorique et sont dépourvus d'un encadrement juridique et de règles de déontologie claires (Igalens, 2009). Les objectifs, les contextes et les utilisateurs de la notation sont spécifiques et souvent peu transparents. Chatterji et al., (2016) 
trouve dans le cadre américain de très faibles corrélations entre les ratings de 6 évaluateurs majeurs de la performance RSE (KLD, ASSET 4, Calvert, FTSE4GOOD, Innovest et DJSI) et suggère de regarder les ratings avec une grande précaution. L'hétérogénéité des méthodologies utilisées, l'opacité des méthodes de notation, l'incertitude liée à de possibles évolutions du référentiel d'évaluation conduisent à poser l'hypothèse suivante.

Hypothèse 1 : Les notes ESG des trois organismes, différent et ne sont pas homogènes.

En effet, si les informations ESG offertes par ces trois organismes sont en majorité destinées aux investisseurs et donc à l'évaluation de la performance, les deux agences «VIGEO et ASSET 4 » dont l'information est un produit de marché et sert de référence à des indices boursiers produisent des données pour toutes les parties prenantes. Au contraire, la société de gestion d'actifs ISR évolue sur un marché concurrentiel et doit fournir une très haute qualité de service pour attirer de la clientèle. Elle devrait être moins influencée par le capital réputation des entreprises qui publient une multitude d'informations pour assurer leur légitimité (Gray, 1995). Les analystes de la société de gestion d'actifs hésitent moins à dégrader les notes, l'objectif premier étant la performance de leurs fonds ISR. La célérité de l'information est primordiale pour que les investisseurs prennent les meilleures décisions (Beaver, 2006). De plus, selon la tétranormalistation, les entreprises, comme les agences, évoluent dans un univers très normalisé conduisant à développer de la conformité (Zardet, Bonnet, 2010 ; Boje et Rosile, 2010). Pour ces organismes, bien installés, le coût afférent à la surévaluation d'une valeur est supérieur au coût d'une sousévaluation (Holthausen et Watts, 2001). Si des divergences d'évaluation sont constatées, elles devraient opposer les deux grandes agences et la société de gestion d'actifs d'où l'hypothèse suivante :

Hypothèse 2 : Compte tenu du conformisme et de la forte normalisation, il existe une homogénéité des notes publiées par les deux grandes agences européennes.

En effet, d'un côté, les notes ESG, compte tenu des objectifs et des critères spécifiques utilisés, notamment l'absence de validation externe du construit et les différences dans l'appréciation de la performance, devraient produire des scores différents (Hypothèse 1), d'un autre côté, le contexte très normalisé du développement de la 
RSE standardise les informations et les données fournies par les entreprises créant un certain conformisme (hypothèse 2) qui incite les organisations à gérer les normes ( Boje, Rosile, 2010, Savall, Zardet, 2005).

Cela étant, les facteurs influençant la notation méritent d'être examinés pour expliquer la variété des résultats. On sait que les travaux antérieurs ont identifié de nombreux éléments explicatifs de la performance sociétale (Malik, 2015 ; Cormier et al., 2011 ; Reverte, 2009, Chih et al., 2010). Vu les différences dans la conception et les attitudes à l'égard du marché entre les pays continentaux, dits à Code de Commerce, et les pays anglo-saxons à dominante de droit coutumier, l'influence du système légal a été prise en compte (La Porta et al., 1998). Le secteur d'activité joue aussi un rôle pour les secteurs risqués (Simnett, 2009, Reverte, 2009). D'après la théorie de la légitimité, la taille de l'entreprise influence l'information fournie par les entreprises (Watts et al., 1986), notamment par les plus grandes qui sont davantage suivies et observées. La dispersion de l'actionnariat favorise par ailleurs l'information volontaire (Johnson et al.,1999 ; Brammer et al., 2008). Enfin, le lien entre performance ESG et performance financière a fait l'objet d'une abondante littérature qui conclut en grande majorité à un effet positif sur la performance financière (Malik, 2015). Des influences positives et négatives de ces éléments devraient s'exercer sur la notation ESG.

\section{2.- La méthodologie}

La recherche analyse les corrélations entre les ratings environnementaux, sociaux et de gouvernance, pour les trois organismes de notation dont les résultats d'évaluation de la performance ESG font l'objet de l'étude.

\subsection{L'échantillon et les variables}

L'échantillon comprend 247 sociétés cotées européennes (dont 66 françaises, 50 allemandes, 21 italiennes, 18 suisses, 19 hollandaises etc.) et 65 sociétés anglo-saxonnes (Angleterre, Irlande, Canada et USA) qui ont fait l'objet d'un rating ESG par les trois organismes. Les données concernent les exercices 2009 à 2012. Les entreprises sont réparties d'après la classification ICB en 5 secteurs 
dont quatre sont identifiés comme risqués (Simnett et al., 2009). Les secteurs plus exposés aux risques sociaux et environnementaux sont : le pétrole-gaz et les matériaux de base, l'industrie et les biens de consommation, les services aux collectivités et les sociétés financières.

L'échantillon est très représentatif. Les sociétés dans le champ comptent parmi les plus grandes capitalisations européennes et américaines mais on trouve également des groupes et des sociétés de taille moindre ce qui fait apparaître une capitalisation moyenne de 22 millions d'euros pour des actifs totaux de 100 millions. Outre la grande diversité de taille, les moyennes respectives de leur rentabilité économique et financière sont de $5 \%$ et $17 \%$.

La méthodologie suivie est en deux étapes. La première est une analyse en composantes principales permettant de réduire les dimensions des données et d'identifier les convergences entre les ratings. La seconde consiste à analyser le lien entre les trois composantes identifiées lors de l'ACP et les déterminants de la performance ESG.

\subsection{Les résultats}

Les résultats de l'ACP font apparaître trois composantes significatives expliquant $67,9 \%$ de la variance totale : pour l'axe 1 , $31,48 \%, 19,57 \%$ pour l'axe 2 ; et $16,87 \%$ pour l'axe 3 (tableau ${ }^{\circ} 2$ ). L'axe 1 reflète la perception de la dimension socioenvironnementale par les deux organismes de notation ASSET 4 et VIGEO. Quatre variables représentatives du rating sont significatives. Leurs analystes ont la même approche du risque social et environnemental et du lien entre ces deux dimensions. La deuxième composante est propre aux trois notes ESG de la société de gestion d'actifs ISR. Les analystes de la société de gestion d'actifs ISR les perçoivent avec une très proche identité de vue. L'axe 3 représente la dimension gouvernance pour VIGEO et ASSET 4. Dans le référentiel des analystes de ces agences, la gouvernance est une composante distincte de la performance sociale et environnementale.

Ainsi, la notation sociale et la notation environnementale de VIGEO et d'ASSET 4 sont très fortement corrélées. La gouvernance est pour eux une catégorie distincte de la performance RSE et leurs ratings de gouvernance sont aussi très convergents. Ils séparent les 
aspects sociaux et environnementaux, des pratiques de gouvernance. En revanche, la société de gestion d'actifs ISR reconnait les critères ESG comme représentatifs d'une seule dimension extra-financière. Leurs analystes apprécient globalement l'information ESG certainement parce qu'ils ne perdent pas de vue leur objectif d'évaluation financière.

Les modèles expliquant les déterminants de la dimension environnementale et sociale (CP1), de la dimension globale ESG (CP2) et de la gouvernance (CP3) issus de l'analyse en composantes principales sont testés séparément à travers des analyses de régression longitudinale. Pour chaque composante, l'équation s'écrit, pour l'observation it : Yit $+1=\alpha+\beta$ Xit $+\varepsilon i, t$. La variable exogène (Yit+1) est successivement la dimension socio-environnementale, la dimension ESG et la dimension gouvernance de l'entreprise i à la période t. Le $\beta$ représente le vecteur des coefficients de régression pour les variables explicatives mesurées par la matrice $X$ et $\varepsilon$ l'aléa. Les variables explicatives sont l'appartenance de l'entreprise à un système juridique, la structure de l'actionnariat, le secteur d'activité, la taille et la performance financière. Les coefficients sont estimés selon un modèle à effets fixes (Baltagi, 2005, Delmas et al., 2013) et les variables explicatives sont décalées d'une année pour que les informations nouvelles aient été intégrées par les investisseurs (Chatterji, 2009, Cho et al., 2016). Deux variables de contrôle sont incluses dans la régression pour limiter l'hétérogénéité des observations, le levier financier (LEV) et la rentabilité économique (ROA).

Le système légal et la taille sont significatifs pour les trois scores de notation mais le sens de la relation est distinct (tableau $\mathrm{n}^{\circ} 3$ ). Les scores de gouvernance d'entreprise sont positivement associés à un fort niveau de protection des investisseurs et négativement à la concentration de l'actionnariat. Les scores sociaux et environnementaux d'ASSET 4 et VIGEO dépendent surtout de la taille, de l'appartenance de l'entreprise à un système de droit civil et du secteur. Le rating ESG de la société de gestion d'actifs est associé à la performance financière, à un système plus souple de protection des investisseurs mais négativement à la taille des entreprises et au risque du secteur.

Il apparait que les facteurs influençant la notation des analystes de VIGEO et ASSET 4 sur le domaine social et environnemental (Modèle 1) sont le secteur d'activité et la taille de l'entreprise, et 
pour les pratiques de gouvernance, la forte protection des actionnaires. Pour le score global ESG de la société de gestion d'actifs ISR, les analystes seraient sensibles à la performance des entreprises opérant dans des systèmes juridiques plus orientés vers les parties prenantes, et à capitalisation plus faible. Pour vérifier la robustesse des résultats, l'analyse a été conduite sans effet de décalage, le sens des résultats obtenus restant identique.

\section{3.- Discussion}

Cette recherche comparative présente l'originalité d'étudier des approches distinctes du rating non financier à travers les résultats des évaluations des performances ESG de trois organismes : celles d'un fournisseur de données ESG brutes ASSET 4, d'un expert européen de notation ESG, VIGEO et d'une société de gestion d'actifs ISR qui utilise ses résultats pour son propre compte. Jusqu'ici, malgré l'importance prise par les publications de reporting non financier pour les investissements et l'information de nombreux stakeholders, peu de comparaisons entre organismes de notation ont été faites (Delmas et al., 2013, Chatterji et al., 2016, Cho et al., 2016). Leur information est donc considérée a priori par les utilisateurs, comme fiable, pertinente et sans biais, bien qu'elle puisse affecter les choix stratégiques et managériaux comme les arbitrages des investisseurs. Or la subjectivité du processus de rating RSE permet de s'interroger sur la validité conceptuelle des mesures obtenues (Sharfman, 1996). Visant un public très large les grandes agences ont un besoin de reconnaissance, laquelle suppose de se conformer à des normes ou règles acceptées (Boje et Rosile, 2010). Cette approche «marché grand public » conduit en fait à une forte corrélation des ratings sur les trois dimensions ESG réduisant de ce fait la diversité suscitée par la recherche d'objectifs spécifiques, contrainte à laquelle échappe la société de gestion d'actifs ISR qui conserve l'information qu'elle traite et ses notations pour atteindre les objectifs de performance boursière de sa clientèle.

La diversité des résultats peut s'expliquer par des différences dans la théorisation de la RSE ou d'objectifs finaux. Ils sont ainsi semblables à ceux de Beaver (2006) mais en partie différents de ceux de Chatterji et al., (2016) qui affichent, dans une étude américaine, de très faibles corrélations entre les ratings de 6 évaluateurs majeurs de la performance RSE (KLD, ASSET 4, 
Calvert, FTSE4, Innovest et DJSI). Faute d'une identité d'approche, les ratings des agences sont à examiner avec prudence.

Les résultats montrent de très fortes corrélations entre les notations sociales et environnementales des deux grandes agences et sur le rating de gouvernance (Adams et al., 1998, Patten, 2002). En revanche, les ratings de la société de gestion d'actifs sont faiblement corrélés aux autres et leurs trois notes ESG représentent une seule dimension. Il existe des différences de perception des critères ESG en fonction des objectifs et des méthodologies utilisées.

L'analyse des facteurs explicatifs des scores montre la conformité des résultats avec les études antérieures : pour la composante environnementale et sociale associées à la taille et au risque du secteur d'activité (Reverte, 2009) pour la dimension globale ESG de la société de gestion d'actifs ISR et la performance financière (Reverte, 2009 ; Chih et al., 2010) et pour la gouvernance $\mathrm{au}$ système juridique en vigueur et à la concentration de l'actionnariat (Graves et al., 1994, La Porta et al., 1998)

Il est à noter que dans les grands organismes de notation, les analystes sont spécialisés par secteurs, parfois par pays, surtout pour l'analyse des pratiques de gouvernance et leur champ d'étude est large. La démarche de la société de gestion d'actifs ISR est plus généraliste et centrée sur l'évaluation. Elle se focalise surtout sur de petites capitalisations boursières. La dimension ESG (composante 2) $\mathrm{y}$ est positivement associée à la performance financière. Au contraire, VIGEO et ASSET 4 offrent de l'information pour la prise de nombreuses décisions autres que purement financières. Leur approche se veut économique, sociale et neutre. Ceci confirmerait la thèse de l'indépendance de la relation entre performance RSE et performance financière avancée par Graves, Waddock (1997) et Mahoney, Roberts (2004).

Conformisme ou pression concurrentielle ? Il se peut que les deux grandes agences de notation ASSET 4 et VIGEO se placent prudemment sur un horizon économique plus long alors que la société de gestion d'actifs se concentre uniquement sur l'utilisation des données dans une optique d'investissement à plus brève échéance (Barth et al., 1994). Dans le référentiel précis des deux agences internationales, la prise en compte des spécificités sectorielles, l'utilisation de grilles plus normatives, conduisent à une vision plus conservatrice, moins directement tournée vers la valorisation, ces agences craignant pour leur réputation l'effet 
pervers d'une surévaluation des titres (Holthausen et al., 2001). La société de gestion d'actifs ISR adopte une démarche directement liée à la performance financière, plus qualitative, pragmatique, sur mesure, permettant, sur le marché concurrentiel de la gestion d'actifs de maintenir un avantage compétitif. Les notes ESG servant au seul usage interne, pour améliorer la performance des portefeuilles de titres, elle peut choisir une approche d'évaluation originale des pratiques ESG, se différenciant de celles des agences commercialisant leurs ratings.

\section{Conclusion}

In fine la recherche, par la comparaison de trois méthodologies de rating ESG, met en évidence des perceptions de la dimension sociale, environnementale et de gouvernance spécifiques pour chaque groupe d'analystes. En l'absence d'une contrainte réglementaire ou législative unique, les mesures servent l'objectif poursuivi et la conception que la société de services se fait des besoins et de l'information à apporter aux utilisateurs qu'elle envisage de satisfaire. Les trois organismes couvrent le même champ et valorisent l'information non financière publiée et volontaire. Les deux grandes agences, le fournisseur de données ESG brutes et l'expert européen de la notation extra financière, sont extrêmement proches. Elles ciblent à la fois les parties prenantes et un segment précis d'investisseurs, qui les utilisent pour l'élaboration d'indices boursiers. La société de gestion d'actifs est centrée sur la rentabilité et la performance des titres à intégrer dans les fonds. Les résultats montrent, contre toute attente, une proximité entre les deux agences internationales qui distinguent les aspects sociaux et environnementaux, des pratiques de gouvernance. La société de gestion d'actifs se différencie et envisage les publications de l'information ESG avec une approche globale cohérente dans un contexte complet d'entreprise orienté vers la création de valeur. Différents objectifs appellent différents scores.

L'apport de cette étude quantitative repose sur l'utilisation d'un même panel représentatif de sociétés cotées de toutes tailles. Elle montre que les notes ESG des grands organismes convergent, ce qui semble être de nature à rassurer leurs utilisateurs, au prix d'une certaine conformité. Elle comporte néanmoins des limites en ce qu'elle étudie les différences de résultats et pas directement les 
processus de notation suivis par les analystes, ce qui serait une autre approche.

En définitive, la prolifération des normes imposées aux entreprises et le choc de conformité y relatif qu'illustre le concept de tétranormalisation, conduit les organismes privés de notation à une homogénéité de résultat et peut-être de comportement.

\section{RÉFÉRENCES BIBLIOGRAPHIQUES}

AdAms C. A., Hill W. Y., Roberts C. B., "Corporate Social Reporting Practices in Western Europe: Legitimating Corporate Behaviour", The British Accounting Review, 1998, 30(1), p.1-21.

BARTH I., "Quand le marketing joue avec la crise des normes in Bessire d., Dupuy y. (coord.), « Tétranormalisation », Cahier de recherche, 2009, ISEOR.

BARTH M. E., McNichols, M. F, "Estimation and market valuation of environmental liabilities relating to superfund sites", Journal of Accounting Research, 1994, 32(3), p.177-209

Beaver W.H, Shakespeare C., Soliman M.T., "Differential properties in the ratings of certified versus non-certified bond-rating agencies", Journal of Accounting and Economics, 2006, 42(3),p.303-334.

Віснот J., Le labyrinthe, "Compliquer pour régner », 2015, Editions Manitoba, Les belles lettres.

Boje L.M., Rosile G. A., « L'éthique et les ailes de la tétranormalisation : le réalisme agentiel », in Bessire d., Cappelletti l., Pigé b. (coord.), Normes : origines et conséquences des crises, éd Economica, coll. « Recherche en gestion », 2010a, Paris.

Brammer S., PAVelin J., "Factors influencing the quality of corporate environmental disclosure", Business Strategy \& the Environment,2008, 17 (2), p.120-136.

CARROLl A.B., «A three dimensional conceptual model of Corporate Social commentary», Business and Society, 33(1), 1979, p. 5-29.

CAPPELLETTI L., « Pratiques esthétiques versus pratiques éthiques de la rse : quelles leçons tirer du cas eads ? », Revue entreprise éthique, dossier spécial : principes éthiques et pratique de la rse, 2008.

ChatterJi A., LeVINe D.P., TofFel, M. W.," How well do social ratings actually measure corporate social responsibility?", Journal of Economics and Management Strategy, 18(1), 2009, p.125-169.

Chatterji A. K., Durand R., Levine D., Touboul S., "Do ratings of firms converge? Implications for strategy research", Strategic Management Journal, ,37(8), 2016, p.1597-1614.

CLARKSON M.B.E., «A stakeholder framework for analysing and evaluating 
corporate social performance», Academy of Management Review, 20(1), 1995, p.92-117.

Cormier D., Ledoux M.J., Magnan M.,"The informational contribution of social and environmental disclosures for investors", Management Decision, 20( 8), 2011, p. 1276-1304.

CHIH, H., CHEN T.,"On the determinants of corporate social responsibility: International evidence on the financial industry", Journal of Business Ethics, 93, 2010, p.115-135.

Cho C., Bouten L., Michelon G., Roberts R., "Does the choice of CSR performance rating provider matter?" Congrès de l'association française de comptabilité, Clermont Ferrand, 2016.

Delmas M. A., EtZion D., NAirn-BirCh N., "Triangulating Environmental Performance: What Do Corporate Social Responsibility Ratings Really Capture?", Academy of Management Perspectives, 27, 2013, p.255-267.

GRAAFLAND J., KAPTEIN M., "The Influence of Ethics Instruction, Religiosity, and Intelligence on Cheating Behavior ", Journal of Business Ethics, 123 (3), 2008, p.437-459.

GRAVES S.B., WADDOCK S., "Institutional owners and corporate social performance", Academy of Management Journal, 37(4), 1994, p.10341047.

Gray R., Kouhy R., Lavers S., "Corporate social and environmental reporting, Accounting", Auditing and Accountability Journal, 8(2), 1995 , p. 47-77.

HolthausEn R., WATt R., "The relevance of value-relevance literature for financial accounting standard setting", Journal of Accounting and Economics 31, 2001, p. 3-75.

IGALENS J., Gond J-P. " Measuring Corporate Social Performance in France: A Critical and Empirical Analysis of ARESE data.", Journal of Business Ethics, 56(2), 2005, p.131-148.

IGALENS J., « Norme de responsabilité et responsabilité des normes : le cas d'iso 26000 », Revue management et avenir, 23, 2009, p.91-104.

ILinitch A. Y., Soderstrom N. S., Thomas T. E., "Measuring corporate environmental performance.", Journal of Accounting and Public Policy, 17, 1998, p383-408.

Jenkins H. M., Yakovleva N., "Corporate Social Responsibility in the Mining Industry: Exploring Trends in Social and Environmental Disclosure", Journal of Cleaner Production 14(3-4), 2006, p.271-284.

La Porta R., Lopez-De-Silanes F., Shleifer A., Vishny R. W., "Law and Finance”, The Journal of Political Economy, 106(6), 1998, p. 1113 1155.

MAHONEY L.S., RoBERTS R.W, « Corporate social performance: empirical evidence on Canadian firms », Research on Professional Responsibility and Ethics in Accounting, 9, 2004, p.73-99.

MAIGNAN I., FERRELl D., "Nature of Corporate Responsibilities : 
Perspectives from American, French and German Consumers", Journal of Business Research, $\mathrm{n}^{\circ} 56,2003$, p. 55-67

MaLiK M., "Value-enhancing capabilities of CSR: A brief review of contemporary literature", Journal of Business Ethics,127, 2015, p 419438.

MARQUEZ. A., Fombrun C. J., "Measuring Corporate Social Responsibility", Corporate Reputation Review, 7(4), 2005, p. 304-308.

Novethic (2014), Panorama des agences de notation extra financières, site NOVETHIC.

ORlitzky M., SchmidT F.L., Rynes S.L., "Corporate Social and Financial Performance: A Meta-Analysis," Organization Studies, 24, 2003, p.403- 441 .

PATTEN D.M., "The relation between environmental performance and environmental disclosure: a research note", Accounting, Organizations and Society, $\mathrm{n}^{\circ} 27,2002$, p. 763-773

REVERTE C., "Determinants of corporate social responsibility disclosure ratings by Spanish listed firms", Journal of Business Ethics, 88, 2009, p.351-366.

Savall H., Zardet V., Tétranormalisation, défis et dynamiques, éd. Economica, Paris, 2005.

Simnett R., Vanstraelen A., Wai Fong Chu A., "Assurance on Sustainability Reports: An International Comparison.”, Accounting Review, 84(3), 2009, p.937-367.

WADDOCK S.A., GRAVES S.B., «The corporate social performance-financial performance link», Strategic Management Journal, 18(4), 1997, p. 303319.

WARTick S.L., Cochran P.L., "The evolution of the corporate social performance in accounting", The Accounting Review, 87(3), 1985, p. $797-806$.

Wood D.J., «Corporate Social Performance Revisited», Academy of Management Review, 16(4), 1991, p. 691-718.

Watts R. L., Zimmerman J. L., Positive Accounting Theory, PrenticeHall, Englewood Cliffs, NJ, 1986.

ZARDET V., BONNET M., Intensification actuelle de la tétranormalisation: risques et bonnes pratiques émergentes », dans Bessire D., Cappelletti L., Pigé B. (coord.), Normes : origines et conséquences des crises, éd. Economica, Paris.2010. 


\section{ANNEXES}

Tableau $n^{\circ} 1$ : Comparaison des méthodes et des référentiels utilisés par les agences (d'après le site internet et adapté de Novethic, 2014)

\begin{tabular}{|c|c|c|c|}
\hline $\begin{array}{l}\text { Sources de } \\
\text { données }\end{array}$ & $\begin{array}{c}\text { Fournisseur de } \\
\text { données brutes } \\
\text { ESG } \\
\text { (ASSET 4) }\end{array}$ & \begin{tabular}{|c|} 
Agence généraliste \\
internationale \\
(VIGEO)
\end{tabular} & $\begin{array}{l}\text { Gérants de fonds } \\
\text { ISR }\end{array}$ \\
\hline Date & 2003 & 2002 & 2008 \\
\hline \multicolumn{4}{|l|}{ Méthodologie } \\
\hline Types de critères & \begin{tabular}{|l} 
Méthodologie \\
d'évaluation \\
intégrée qui repose \\
sur quatre piliers : \\
18 catégories, 250 \\
indicateurs de \\
performance, 750 \\
points de données.
\end{tabular} & \begin{tabular}{|l|}
38 enjeux, 6 \\
domaines. \\
Evaluation de la \\
pertinence des \\
engagements et de \\
leur mise en cuvve, \\
des résultats \\
obtenus.
\end{tabular} & $\begin{array}{l}\text { La performance } \\
\text { des entreprises } \\
\text { notée selon trois } \\
\text { piliers répartis en } \\
18 \text { dimensions. } 80 \\
\text { critères }\end{array}$ \\
\hline $\begin{array}{l}\text { Source } \\
\text { d'informations }\end{array}$ & $\begin{array}{l}\text { Données publiques } \\
\text { collectées: } \\
\text { entreprises, ONG et } \\
\text { presse. }\end{array}$ & $\begin{array}{l}\text { Collecte multi } \\
\text { sources Données } \\
\text { publiques, enquêtes, } \\
\text { rencontres. }\end{array}$ & $\begin{array}{l}\text { Collecte multi } \\
\text { sources, Données } \\
\text { publiques, } \\
\text { enquêtes, } \\
\text { rencontres, visites. }\end{array}$ \\
\hline Sous-traitance & $\begin{array}{l}\text { Analystes } \\
\text { délocalisés }\end{array}$ & Analystes locaux & Analystes locaux \\
\hline Transparence & $\begin{array}{l}\text { Oui, Notation } \\
\text { divulguée mais } \\
\text { payante. }\end{array}$ & $\begin{array}{l}\text { Oui. Notation } \\
\text { divulguée mais } \\
\text { payante. }\end{array}$ & $\begin{array}{l}\text { Non. Notation } \\
\text { ESG à usage } \\
\text { interne, pour la } \\
\text { sélection des fonds } \\
\text { gérés. }\end{array}$ \\
\hline Objectifs & $\begin{array}{l}\text { Information destinée } \\
\text { aux investisseurs et } \\
\text { parties prenantes. }\end{array}$ & $\begin{array}{l}\text { Information } \\
\text { destinée aux } \\
\text { investisseurs et } \\
\text { parties prenantes. }\end{array}$ & $\begin{array}{l}\text { Sélection des } \\
\text { valeurs à inclure } \\
\text { dans les fonds. }\end{array}$ \\
\hline \multicolumn{4}{|c|}{ Caractéristiques des informations } \\
\hline $\begin{array}{l}\text { Analyse } \\
\text { normative }\end{array}$ & non & oui & oui \\
\hline Indices ISR & Oui (8 indices) & Oui (8 indices) & (fonds ISR) \\
\hline N. analystes & 130 & 80 & 4 \\
\hline Nombre ent. & 4000 mondiales & 2800 mondiales & $\begin{array}{l}700 \text { européennes et } \\
\text { anglo-saxonnes }\end{array}$ \\
\hline $\begin{array}{l}\text { Méthode de } \\
\text { travail }\end{array}$ & $\begin{array}{l}\text { Répartition des } \\
\text { analystes par secteur }\end{array}$ & $\begin{array}{l}\text { Répartition des } \\
\text { analystes par } \\
\text { secteur }\end{array}$ & $\begin{array}{l}\text { Analystes } \\
\text { généralistes et } \\
\text { gérants de fonds }\end{array}$ \\
\hline
\end{tabular}


Tableau n ${ }^{\circ} 2$ : Résultat de l'ACP sur les notes ESG des trois organismes

\begin{tabular}{|c|c|c|c|c|}
\hline \multirow[t]{2}{*}{$\mathrm{ACP}$ - } & \multicolumn{4}{|c|}{ Composantes (Rotation varimax) } \\
\hline & & Correlation & Correlation & Corr. \\
\hline Source des données & Score & 1 & 2 & 3 \\
\hline ASSET 4 & AENV & 0,801 & 0,007 & 0,186 \\
\hline ASSET 4 & $\mathrm{ASOC}$ & 0,818 & 0,124 & 0,161 \\
\hline ASSET 4 & AGOV & 0,253 & 0,036 & 0,789 \\
\hline VIGEO & BENV & 0,781 & 0,096 & 0,207 \\
\hline VIGEO & $\mathrm{BSOC}$ & 0,799 & 0,001 & $-0,096$ \\
\hline VIGEO & BGOV & 0,034 & $-0,045$ & 0,864 \\
\hline SOC ISR & CFNV & 0,417 & 0,729 & 0,002 \\
\hline SOC TSR & $\mathrm{CSOC}$ & 0,085 & 0,781 & $-0,139$ \\
\hline SOC ISR & CGOV & $-0,167$ & 0,769 & 0,137 \\
\hline valeur propre (eigenvalue) & & 2,833 & 1,761 & 1,518 \\
\hline Variance expliquée & & $31,48 \%$ & $19,57 \%$ & $16,87 \%$ \\
\hline Variance expliquée cumulée & & $31,48 \%$ & $51,04 \%$ & $67,9 \%$ \\
\hline
\end{tabular}


Tableau n $\mathbf{3}$ : Résultat des régressions longitudinales

\begin{tabular}{|c|c|c|c|c|c|c|}
\hline \multirow[t]{2}{*}{$\begin{array}{l}2009-2012 \\
\mathrm{~N}=623 \text { obs }\end{array}$} & \multicolumn{2}{|c|}{$\begin{array}{c}\text { Modèle CP1 } \\
\text { (ratings env et soc, } \\
\text { ASSET } 4 \text { et } \\
\text { VIGEO) }\end{array}$} & \multicolumn{2}{|c|}{$\begin{array}{c}\text { Modèle CP2 : ESG } \\
\text { ( Société de gestion } \\
\text { ISR) }\end{array}$} & \multicolumn{2}{|c|}{$\begin{array}{c}\text { Modèle 3 : } \\
\text { Gouvernance(ASSET } \\
4 \text { et VIGEO) }\end{array}$} \\
\hline & coeff & t & coeff & $t$ & coeff & $\mathbf{t}$ \\
\hline Const. & -5.03 & $-1.9 * *$ & 1.530 & $3.55 * * *$ & -1.088 & $-3.0 * * *$ \\
\hline Common & $-0,627$ & $-6 . * * *$ & -0.36 & $-3.2 * * *$ & 1.272 & $13.4 * * *$ \\
\hline Act & 0.138 & $1.98 * *$ & -0.024 & -0.31 & -0.573 & $-8.7 * * *$ \\
\hline Sect & 0.154 & $1.96 * *$ & -0.186 & $-2,11 * *$ & 0.0672 & 0.91 \\
\hline $\mathrm{MtB}$ & -0.01 & -1.75 & 0.04 & 4.55 *** & -0.003 & -0.44 \\
\hline Roa & 0.851 & 1.36 & 0.592 & 0.85 & 0.351 & 0.60 \\
\hline Lev & -0.011 & -1.00 & -0.02 & -1.64 & -0.000 & -0.03 \\
\hline $\ln \mathrm{TA}$ & 0.292 & $13.2 * * *$ & -0.085 & $-3.4 * * *$ & -1.088 & $3.22 * * *$ \\
\hline $\mathrm{R}^{2}$ & \multicolumn{2}{|c|}{$\frac{1}{30.31 \%}$} & \multicolumn{2}{|c|}{$\frac{1}{10.2 \%}$} & \multicolumn{2}{|c|}{$38.4 \%$} \\
\hline Fisher & \multicolumn{2}{|c|}{$38 * * *$} & \multicolumn{2}{|c|}{$9.94 * * *$} & \multicolumn{2}{|c|}{$53.7 * * *$} \\
\hline
\end{tabular}


Tableau ${ }^{\circ} \mathbf{4}$ : Description des variables indépendantes

\begin{tabular}{|c|c|}
\hline & Description \\
\hline COMMON & $\begin{array}{l}\text { "Common law » est une variable dichotomique qui prend la valeur } 1 \text { si le pays est d'origine anglo-saxonne } \\
\text { ( USA, UK, Canada) et } 0 \text { sinon ( La Porta et al.,1998). }\end{array}$ \\
\hline SECTEUR & $\begin{array}{l}\text { Est une variable dichotomique qui prend la valeur } 1 \text { si le secteur présente un risque environnemental et } \\
\text { social, } 0 \text { sinon.( Simnett et } \\
\text { al., (2009). }\end{array}$ \\
\hline Ln TA & Le logarithme népérien de l'actif total. \\
\hline ACT & $\begin{array}{l}\text { La structure de l'actionnariat est une variable dichotomique prenant la valeur } 1 \text { quand un groupe } \\
\text { d'investisseurs détient plus } \\
\text { de } 15 \% \text { de l'actionnariat, } 0 \text { sinon (Cormier et al., 2011). }\end{array}$ \\
\hline MTB & $\begin{array}{l}\text { Le market to book est le rapport entre la valeur de marché des fonds propres en fin d'année et la valeur } \\
\text { comptable des fonds } \\
\text { propres. }\end{array}$ \\
\hline
\end{tabular}

Nota : la liste exhaustive des sociétés et groupes compris dans l'échantillon est disponible sur demande. 